\title{
Investigating high-mass star formation through maser surveys
}

\author{
S. P. Ellingsen ${ }^{1}$, M. A. Voronkov ${ }^{2}$, D. M. Cragg ${ }^{3}$, A. M. Sobolev ${ }^{4}$, \\ S. L. Breen ${ }^{1}$ and P. D. Godfrey ${ }^{3}$ \\ ${ }^{1}$ School of Mathematics and Physics, University of Tasmania, Private Bag 21, Hobart 7001, \\ TAS, Australia \\ email: Simon.Ellingsen@utas.edu.au \\ ${ }^{2}$ Australia Telescope National Facility CSIRO, PO Box 76, Epping 1710, NSW, Australia \\ ${ }^{3}$ School of Chemistry, Building 23, Monash University, Victoria 3800, Australia \\ ${ }^{4}$ Ural State University, Lenin ave. 51, 620083 Ekaterinburg, Russia
}

\begin{abstract}
Interstellar masers are unique probes of the environments in which they arise. In studies of high-mass star formation their primary function has been as signposts of these regions and they have been used as probes of the kinematics and physical conditions in only a few sources. With a few notable exceptions, we know relatively little about the evolutionary phase the different maser species trace, nor their location with respect to other star formation tracers. While detailed studies of a small number of maser regions can reveal much about them, other information can only be obtained through large, systematic searches. In particular, such surveys are vital in efforts to determine an evolutionary sequence for the common maser species, and there is growing evidence that methanol masers may trace an earlier phase than the other common maser species of $\mathrm{OH}$ and water.
\end{abstract}

Keywords. stars: formation, Masers, Surveys

\section{Introduction}

Interstellar masers are one of the best, if not the best signpost of young high-mass star formation regions. They are relatively common, intense and because they arise at centimetre wavelengths, are not affected by the high extinction that plagues observations in other wavelength ranges. The $6.7 \mathrm{GHz}$ transition of methanol has been found to be a particularly useful signpost as it is both common and strong and appears to only be associated with high-mass star formation regions (Minier et al. 2003). The methanol multibeam (MMB) survey currently being undertaken with the Parkes and Jodrell Bank telescopes is using observations of the $6.7 \mathrm{GHz}$ transition of methanol to trace the Galactic distribution of star formation and investigate the structure of our Galaxy (see the papers by Green et al. and Pestalozzi et al. in these proceedings).

In addition to being excellent signposts of star formation regions, masers have characteristics that make them very well suited to be utilised as probes of the regions. The maser emission is compact and intense, which means that it can be studied at milliarcsecond resolution using very long baseline interferometry (VLBI) techniques. This makes masers ideal probes of the kinematics of the gas in which they arise (e.g. Torrelles et al. 2005). They are potentially equally powerful probes of the physical conditions in these same regions (e.g. Cragg et al. 2001), however, the complexity of maser pumping schemes provides significant challenges for robust interpretation of the results. Although progress has been slow towards the overall goal of utilizing masers as tools to study star formation, the pace of advance has recently accelerated. The proliferation of complementary 
high-resolution observations of star formation regions at millimetre through mid-infrared wavelengths means that this trend is likely to continue.

Some of the questions relating to masers are best addressed through detailed studies of many transitions, molecules and wavelength bands towards a small number of sources. For example this is the best means of determining where the different maser species lie with respect to other objects within star formation regions. However, favourite sources which show strong emission from a wide range of maser transitions are atypical of high-mass star formation regions and so there are some questions for which a statistical approach is a better way to proceed. Uncommon phenomena must be associated with unusual sources, or brief evolutionary phases. What we really want to know are the common properties of masers in star formation regions, as they can give us an insight into the general characteristics of the process.

\section{An Evolutionary Sequence for Masers}

There are four species of interstellar maser which are common in high-mass star formation regions. Mainline $\mathrm{OH}$ masers at $1.6 \mathrm{GHz}$ and water masers at $22 \mathrm{GHz}$ have been studied since the 1960s, while the 1980s and 1990s saw the discovery of a number of strong transitions from two different types of methanol masers. The $\mathrm{OH}$, water and class II methanol masers are found close to newly formed high-mass stars, as indicated by the presence of Hir regions and bright infrared sources. The class I methanol masers are found towards high-mass star formation regions, but are usually offset from the highmass protostars and other maser species. The presence of an interstellar maser within a star formation region signifies "special" physical conditions. They are special in the sense that masing is only predicted to occur for certain ranges of temperature, density, molecular abundance etc. Theoretical models find the most common maser species mainline $\mathrm{OH}, 6.7 \mathrm{GHz}$ methanol and $22 \mathrm{GHz}$ water masers are inverted over a wide range of physical conditions (e.g. Cragg, Sobolev \& Godfrey 2002).

At present there remains significant uncertainty about where within star formation regions the different maser species arise and the evolutionary phase they are associated with. However, as relatively easily observed signposts of star formation regions, it would be very desirable to be able to use the presence or absence of the different maser species as an evolutionary clock. Towards many star formation regions more than one (sometimes all) of the common maser species are observed, which implies that there is significant overlap between the evolutionary phases traced. The complexity of the goal of finding an evolutionary sequence for masers in high-mass star formation regions should not be underestimated, indeed De Buizer et al. (2005) suggest that the different maser species trace a variety of stellar phenomena and are hence associated with multiple evolutionary phases. It is only through statistical studies of large samples of maser sources that a definitive answer will be obtained.

There are a few facts that are commonly agreed for the common maser species - in the words of former US Secretary of Defense Donald Rumsfeld "Things we know we know" :

(a) Water masers usually trace outflows (e.g. Felli, Palagi \& Tofani 1992).

(b) Water masers trace a generally earlier evolutionary phase than $\mathrm{OH}$ masers (Forster \& Caswell 1989).

(c) Where $\mathrm{OH}$ and methanol masers are observed towards the same regions the clusters arise from the same general region (Caswell 1997), although there is no coincidence between the individual maser spots of the different species (Menten et al. 1992).

A number of independent lines of evidence suggest that in many cases the class II methanol masers are associated with a very early stage of the high-mass star formation 
process. In particular, only a small percentage are associated with ultra-compact HiI regions (Phillips et al. 1998, Walsh et al. 1998), but all are associated with millimetre (Pestalozzi, Humphreys \& Booth 2002) or submillimetre sources (Walsh et al. 2003) and many are embedded within infrared dark clouds (Ellingsen 2006). They are associated with sources which have a spectral energy distribution consistent with them being deeply embedded (Minier et al. 2005). This raises the obvious question, are some of the class II methanol masers associated with an earlier phase of high-mass star formation than water masers?

\subsection{Water and Class II methanol}

There have been a small number of comparative studies of water and class II methanol masers. Sridharan et al. (2002) searched for water and methanol masers towards an infrared-based sample of candidate high-mass star formation regions and found that the majority of sources which had associated masers showed emission from both species. While a search for water masers towards a sample of methanol masers by Szymczak, Pillai \& Menten (2005) achieved a detection rate of approximately $50 \%$. These studies suggest that water and methanol masers are commonly observed towards the same star formation regions, however, targeted searches inevitably suffer potential biases arising from the selection criteria. A recent complete survey for water maser emission towards the G333.6-0.2 giant molecular cloud (Breen et al. 2007) complements previous unbiased surveys of $6.7 \mathrm{GHz}$ methanol (Ellingsen et al. 1996) and main-line OH masers (Caswell, Haynes \& Goss 1980) in the same region. For the first time we have been able to compare in detail the distribution of these different maser species over an area of more than a square degree. Figure 3 of Breen et al. shows a striking dichotomy between the water and methanol masers in the region. All the water masers lie very close to the main axis of the cloud, as traced by the bright mid-infrared emission, while the methanol masers generally lie near the interface between the stronger and more diffuse emission.

That the water masers are generally projected against bright mid infrared emission along the main axis of the region suggests that they are associated with relatively evolved high-mass star formation. The presence of methanol masers offset from the brightest midinfrared emission and their absence along the main axis suggests that they may be tracing a second epoch of star formation in the region, triggered by energetic winds and outflows from the mid infrared bright sources. The water maser search of Breen et al. was only sensitive to water masers with a peak flux density stronger than 5 Jy and searched a smaller fraction of the giant molecular cloud than the methanol and $\mathrm{OH}$ searches, so it is likely that there are some weak water maser sources in the region that have not yet been detected. It is not possible to draw general conclusions on the basis of observations of a single giant molecular cloud, however, the results strongly suggest that $6.7 \mathrm{GHz}$ methanol masers trace an earlier evolutionary phase of high-mass star formation than do luminous water masers.

\subsection{Class I and class II methanol}

Class I methanol masers are the least well understood of the common maser species. They occur at the interface between outflows and the ambient molecular cloud environment (Plambeck \& Menten 1990), offset from the other maser species. One of the main difficulties in studying class I methanol masers has been the lack of good criteria on which to base targeted searches. Ellingsen (2005) showed that approximately $40 \%$ of class II methanol masers have an associated class I methanol maser within 30 arcseconds. Recent ATCA observations have shown that in many cases the class I maser sources are not merely chance detections towards other objects within the larger star formation regions, 


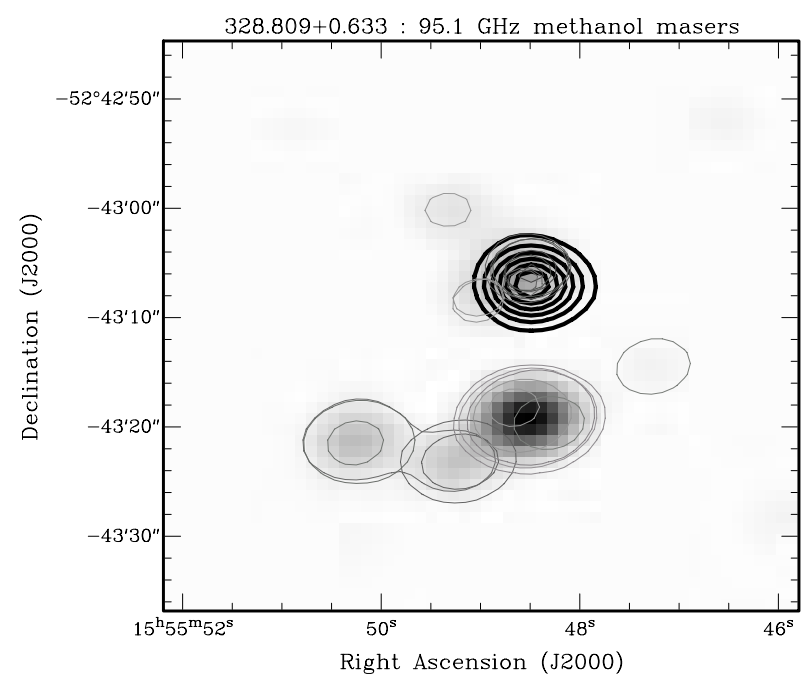

Figure 1. G328.81 + 0.63 shows strong (400 Jy) class II methanol maser emission projected against the ultra-compact HiI region (thick black contours - centred at approximately $\alpha=15: 55: 48.5, \delta=-52: 43: 07)$. The greyscale image shows the integrated $95.1 \mathrm{GHz}$ class I methanol maser emission and the thin grey contours are a renzogram representing the velocity of the emission.

but are intimately associated with the class II masers. Figure 1 shows the $95.1 \mathrm{GHz}$ class I methanol masers towards the well known class II methanol maser source G328.81+0.63. The class I masers are spread over 8 or more clusters covering an extent of more than 30 arcseconds, the strongest emission being to the south of the HiI region. In contrast the class II methanol masers lie within a single cluster projected against the north-east of the HII region. There is class I methanol maser emission from the same projected location as the class II masers and encompassing the same velocity range. Theoretically strong class I and class II methanol masers cannot coexist - the former are collisionally excited, while the latter are radiatively pumped. It may be that the class I maser are associated with an outflow directed nearly perpendicular to the line of sight from the source pumping the class II masers. Regardless of the detailed interpretation, these and other ATCA observations demonstrate that in many cases class I and class II methanol masers are associated with the same driving source, rather than a coincidental association.

The presence of molecular outflows is thought to be one of the earliest indications of the commencement of star formation and so we might naively expect class I masers to trace an even earlier evolutionary phase than either water or class II methanol masers. Ellingsen (2006) investigated the mid infrared colours (from the GLIMPSE survey) of a sample of class II methanol masers with and without associated class I methanol masers. Although the sample is relatively small, there is a strong tendency for the sources with an associated class I methanol maser to have redder colours. Redder colours are normally associated with more deeply embedded, and hence younger sources. 


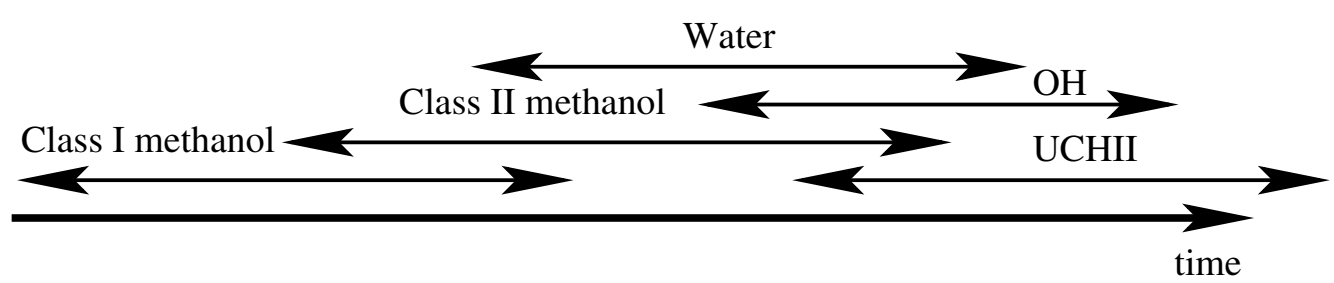

Figure 2. A "straw man" evolutionary sequence for masers in high-mass star formation regions.

\section{Conclusions}

Figure 2 outlines a "straw man" evolutionary sequence for masers in star formation regions that is consistent with the results outlined above and previously established facts. This sequence appears consistent with the general properties of masers in highmass star formation regions, however, there are specific sources which are not consistent with this sequence. For example the Turner-Welsh object near W3(OH) has associated water and $\mathrm{OH}$ maser emission, but no methanol masers (Argon, Reid \& Menten 2003), while IRAS16547-4247 has class I methanol, water and $\mathrm{OH}$, but no class II methanol masers (Voronkov et al. 2006). These may be rare sources, but only rigorous statistical testing will show whether the proposed sequence usefully describes the majority of cases.

\section{References}

Argon, A. L., Reid, M. J., \& Menten, K. M. 2003 ApJ 593, 925

Breen, S. L., Ellingsen, S. P., Johnston-Hollitt, M., Wotherspoon, S., Burton, M. G., Cunningham, M., Lo, N., Senkbeil, C. E., \& Wong, T. 2007 MNRAS in press

Caswell, J. L. 1997 MNRAS 289, 203

Caswell, J. L., Haynes, R. F., \& Goss, W. M. 1980 Aust. J. Phys. 33, 639

Cragg, D. M., Sobolev, A. M., Ellingsen, S. P., Caswell, J. L., Godfrey, P. D., Salii, S. V., \& Dodson, R. G. 2001 MNRAS 323, 939

Cragg, D. M., Sobolev, A. M., \& Godfrey, P. D. 2002 MNRAS 331, 521

De Buizer, J. M., Radomski, J. T., Telesco, C. M., \& Piña, R. K. 2005 ApJS 156, 179

Ellingsen, S. P. 2005 MNRAS 359, 1498

Ellingsen, S. P. 2006, ApJ 638, 241

Ellingsen, S. P., von Bibra, M. L., McCulloch, P. M., Norris, R. P., Deshpande, A. A., \& Phillips, C. J. $1996, M N R A S 280,378$

Felli, M., Palagi, F., \& Tofani, G. 1992, A\& A 255, 293

Forster, J. R., \& Caswell, J. L. 1989, A\&̈A 213, 339

Menten, K. M., Reid, M. J., Pratap, P., Moran, J. M., \& Wilson, T. L. 1992, ApJ 401, L39

Minier, V., Burton, M.G., Hill, T., Pestalozzi, M.R., Purcell, C.R., Garay, G., Walsh, A.J., \& Longmore, S. 2005 A $\& A 429,945$

Minier, V., Ellingsen, S. P., Norris, R. P., \& Booth, R. S. 2003, A\&3A 403, 1095

Pestalozzi, M. R., Humphreys, E. M. L., \& Booth, R. S. 2002 A\&A 384, L15

Phillips, C. J., Norris, R. P., Ellingsen, S. P., \& McCulloch, P. M. 1998, MNRAS 300, 1131

Plambeck, R. L., Menten, K. M. 1990 ApJ 364, 555

Sridharan, T. K., Beuther, H., Schilke, P., Menten, K. M., \& Wyrowski, F. 2002, ApJ 566, 931

Szymczak, M., Pillai, T., \& Menten, K. M. 2005, A $\& A$ 434, 613

Torrelles, J. M., Patel, N., Gómez, J.F., Anglada, G., \& Uscanga, L. 2005, Ap\&SS 295, 53

Voronkov, M. A., Brooks, K. J., Sobolev, A. M., Ellingsen, S. P., Ostrovskii, A. B., \& Caswell, J. L. 2006 MNRAS 373, 411

Walsh, A. J., Burton, M. G., Hyland, A. R., \& Robinson, G. 1998 MNRAS 301, 640

Walsh, A. J., Macdonald, G. H., Alvey, N. D. S., Burton, M. G., \& Lee, J.-K. 2003 A\&A 410, 597 